\title{
TARGETED INTERVENTION SERVICES FOR PEOPLE WHO INJECT DRUGS IN NEPAL
}

\author{
Dipendra Bahadur K.C. *
}

\section{ABSTRACT}

This study was conducted in the several districts of TI project implemented areas where 102 respondents as a sample size and 12 were the Female Who Injects Drugs (FWID): Kathmandu, Lalitpur Kaski, Tanahu, Chitwan, Kailali, Sarlahi and Jhapa respectively. For the study 10-15 participants enrolled from each districts. The major findings of the study are based upon the Drug User's perspectives includes: PWIDs were unable to receive services from the Dropping in Centers (DIC) due to the extreme police harassment in the Kaski district. Using contaminated syringe, sharing of used syringe in a group, stolen syringe from the hospitals were the common trend identified during the gap of the project for PWIDs by Nepal Government in Kaski district, Tanahu district and Chitwan district respectively. This study reveals that there was lack of standard intervention protocol and guidelines for the PWIDs community. Furthermore, comprehensive package as well as multi years project was felt to be necessary without any gaps in the services to reduce the HIV transmission, HCV and Hep B among the PWIDs.

Keywords: NCASC (National Centre for Aids and STD Control), Stigma \& discrimination, Police harassment, Service gap, needle \& Syringe, HIV, Blood borne disease, Nepal Government, primary Health Care(PHC), Drop in Centre (DIC), Project, Problem solving.

\section{INTRODUCTION}

People Who Inject Drugs are among the group most vulnerable to HIV infection. HIV prevalence among injecting Drug Users was 28 times higher than among the rest of the population (Harm Reduction International, 2016). The alarming problem of PWIDs cannot be efficiently overcome unless the various agencies such as governmental and nongovernmental organizations in the different disciplines are prepared to co-operate and coordinate depending upon the aspects in which they can become most

* Mr. K.C. is a Lecturer, Padma Kanya Multiple Campus, Bagbazar, TU. 
effective. The result of the study were expected to inform service providers, policy makers and to improve resource allocation for the continuation and the sustainability of the project for the benefits of the PWIDs to lowered the transmission of HIV rate. The Central Bureau of Statics (CBS) in collaboration with the Ministry of Home affairs conducted a survey on current hard drug users in Nepal in 2012/2013. The survey report published in 2013 revealed 91,534 current hard drug users in Nepal, which was nearly double of 46,309 in 2006 (CBS 2006). A study conducted by NCASC in coordination with other relevant organization regarding IBBS survey tried to find out the prevalence, trend and situation of PWIDs. The findings of the study showed the prevalence rate of HIV, STI and Hep B was significant among PWIDs. The prevalence rate of HIV was high among PWIDs in the study conducted districts. (NCASC, 2016/17).

Sharing needles, syringes, and other injection equipment was a direct route of HIV transmission. In the recent targeted districts Kathmandu and Lalitpur where the PWIDs population was high in compare to other districts. Recent report of NAMUNA (till June 2018), more than 900 PWIDs identified as a new client in both of the districts. Moreover, in other districts also the PWIDs population was high. The major concerned of this study was that if there will be gaps in the services certainly there will be the higher chances of the growing HIV infection rate among the PWIDs in Nepal. Such gap in the services directly hampers the national goal on HIV prevention among the PWIDs. Hence, the research tried to understand about what was the actual scenario among PWIDs during the gap of the project?

This study will contribute largely to the Government of Nepal, National center for AIDS and STD control (NCASC), Targeted Intervention program for PWID in Nepal, policy makers and planner of different NGO and INGO working on the Harm Reduction in Nepal.

\section{OBJECTIVES}

The objectives of the study are:

- To identify actual scenario among the PWIDs during the gap of the project. 
- To analyze the Macro level social work intervention and its gap between targeted group and service providers.

- To access various problems as faced by PWIDs in the study area.

\section{REVIEW OF LITERATURE}

A study conducted by NCASC in coordination with other relevant organization regarding IBBS survey tried to find out the prevalence, trend and situation of PWIDs. A survey conducted for those PWIDs/FWIDs above the age of 16 and used injecting drugs prior three months of the survey. Data of the survey disclosed by press release of the year 2074/2075 B.S. The result of the study was given below:

Table 1: Prevalence, trend and Situation of PWIDs

\begin{tabular}{|c|c|c|c|c|c|}
\hline Regions & 言 & 至 & 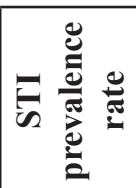 & 总 & 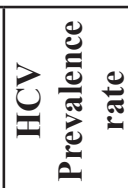 \\
\hline $\begin{array}{l}\text { Male (PWIDs) eastern region( } \\
3 \text { districts) }\end{array}$ & 360 & $3.3 \%$ & $1.9 \%$ & $0.8 \%$ & $38.1 \%$ \\
\hline $\begin{array}{l}\text { Male(PWIDs) Western to far } \\
\text { western region( } 7 \text { districts) }\end{array}$ & 300 & $5.3 \%$ & $2.0 \%$ & $2.7 \%$ & $23.7 \%$ \\
\hline $\begin{array}{lll}\begin{array}{l}\text { Female } \\
\text { valley) }\end{array} & \text { (FWIDs) } & \text { (Pokhara } \\
\end{array}$ & 155 & $1.9 \%$ & $3.9 \%$ & $1.3 \%$ & $2.6 \%$ \\
\hline
\end{tabular}

Source: NCASC, 2016/17.

The findings of the study had indicated the prevalence rate of HIV, STI and Hep B and HCV was significant among PWIDs. Moreover, prevalence rate of $\mathrm{HCV}$ was high among PWIDs.

Drug Control policy 2063, included that due to urbanization and the major cities like Kathmandu, Pokhara, Lalitpur, Dharan and in the open border areas the use of drug was very high then the other areas of Nepal. In the policy, 5.3.1 article clearly mentioned on the health issues of the drug user's. Likewise in the article 5.3.2 to reduce HIV transmission there should be implementation of. HIV prevention programs. (Drug Control Policy, 2063). 
In Nepal there was HIV policy for PWIDs but contradicts with drug control law, which created problems for effective implementation for HIV prevention programs. Thus there was a need of specific protocol and guidelines for PWIDs community. Due to lack of policies for the PWIDs were isolated and in some cases deprived from the right to services. There has been found that lack of standard intervention protocol guidelines for PWIDs community. Even the donor agencies and the relevant organizations were unable to recognize the threat for the PWIDs in the transmission of the HIV. Furthermore, there was huge programmatic gap specifically for the FWIDs and there was no access to abscess management, Hep C and Hep B regarding the PWIDs community. Furthermore, Once used needle and syringe was thrown anywhere in the community by the PWIDs, which was harmful to children who plays at that place and towards the community people who walks around those areas was major challenge towards the community people. Corrigan (2005), Social workers bring a strengths approach to the multidisciplinary Field. By focusing on clients' strengths, Social Workers can counter the stigma and discrimination that occurs when the individual's diagnosis becomes an enduring label. Likewise, NCASC (2010) indicated that stigma and discrimination has been identified among the FWIDs by the community thus FWIDs are deprived from their rights to services was also challenging situation for the FWIDs in Nepal.

\section{METHODOLOGY AND SOURCES OF DATA}

This study focused on the problem solving methods. Perlman, 1957 as advocated the problem solving method of intervention. Her goal was to move away from the psychosocial focus on early childhood experiences and memories and to make social work practice on the present moment. The findings of the study based upon the Drug User's perspectives. This study had been conducted in the following districts: Kathmandu district, Lalitpur district, Chitwan district, Kaski district, Tanahu district, Sarlahi district, Kailali district and Jhapa district respectively. The total no. of sampling size was 102 where 12 respondents were FIDUs. The researcher has used qualitative data procedures. For the study respondents selected those who were enrolled in the previous TI project till now. To understand the actual scenario among the PWIDs during the gap of the project such PWIDs selected for the Study. From each of the district's 10-15 sampling size 
selected for the study. Included districts are Kathmandu, Lalitpur, Chitwan, Kaski, Tanahu, Sarlahi, Kailali and Jhapa selected respectively.

For the study open ended questions were developed to identify the depth knowledge of the area under research considerations. The questionnaire were developed for both the enrolled clients and the staffs of the DICs. Regular field visit and organizational visits were initiated for the study to achieve relevant data and information. The major tools applied for the study was the FGD to identify the actual scenario among the PWIDs. The participants FGD was convinced to keep their names confidential. Case study conducted to obtain extend narratives and descriptions of the high risk behavior among the PWIDs during the gap of the project and the success story while receiving the services previously. Thus this study included the two case studies among the PWIDs. Interview conducted during the field visit among the PWIDs and the staffs in the DICs. Interview initiated to gain the subjective understanding and to achieve the goal of the study.

\section{ANALYSIS}

Whether in the gap of the project or ongoing of the project PWIDs were facing police harassment problems that enforces to live their life with insecurities and tensions. In the gap of the project irregular health checks up and they enforce to face stigma and discrimination around their environment. That causes low self-esteem and unable to take right decisions in their life. Police harassment was one of the common problems among the PWIDs found in all the districts. Another common problem was the stigma and discrimination towards the PWIDs in all the districts. Hanging with the friends and spending time in high risk zone during the gap of the project. "Even it's hard to spend time in DIC due to police harassment". Continuation of the project was the most essential for the PWIDs to spend their time without drug related harms. PWIDs can spend their time in the DICs and can receive services and counseling through the DICs.

In the gap of the project sharing syringe in the group was the most common trend among the PWIDs. In some cases they stole syringe from the hospital and are motivated to use contaminated syringe again and again. "In Kaski district some of the PWIDs stolen syringe from the hospital and used contaminated syringe from the garbage of the hospital. Even in Chitwan and Tanahu district PWIDs were using contaminated syringe and using single 
syringe again and again that increase HIV infections rate highly among the PWIDs and their partners". Stigma and discrimination was one of the major challenge PWIDs faces in their daily life. In most of the districts PWIDs were aware about the transmission of the HIV but most of the PWIDs were still unaware about the HIV, HeB in all the districts. In the Jhapa district and Sarlahi districts PWIDs shared single syringe in the group. Thus, sharing syringe in a group and using contaminated syringe among the PWIDs were the most common trend during the gap of the project and in all the districts there was maximum chances of the transmission of the HIV infections and other blood borne diseases.

Table 2: District wise HTC Service availability

\begin{tabular}{|l|c|c|c|c|}
\hline Districts & \multicolumn{4}{|c|}{ Services in the Districts } \\
\hline & HTC & OST & REHAB & PHC \\
\hline Tanahu & Access & No Access & No Access & No Access \\
\hline Kaski & Access & Access & No Access & No Access \\
\hline Kathmandu & Access & Access & No Access & No Access \\
\hline Lalitpur & Access & Access & No Access & No Access \\
\hline Jhapa & Access & No Access & No Access & Access \\
\hline Chitwan & Access & Access & No Access & Access \\
\hline Sarlahi & Access & No Access & No Access & No Access \\
\hline Kailali & Access & No access & No Access & No Access \\
\hline
\end{tabular}

Source: Field survey, 2018

In the above table HTC services were easy access to the PWIDs from the relevant organization and from the district Hospitals. In Jhapa and Chitwan District for PHC services PWIDs were referral to Hospital but there was no any free of cost for the PHC services. In all the districts there was no any free treatment for rehab procedure thus no easy access for PWIDs for the Rehab. In Chitwan, Lalitpur, Kathmandu and Kaski there was access to OST for the PWIDs.

For the effectiveness of the project there was need of the long term project. Only HTC testing was not enough to tackle the problems of the PWIDs there also required of HIV testing and treatment in the DICs. There was requirement of the comprehensive services to achieve the goal of the project. Furthermore, there should be the requirement of the proper PHC 
services. There should not be any police harassment towards the PWIDs who were enrolled in the DICs. "The recent services were not qualitative In comparison to the previous services". To reduce stigma and discrimination awareness program was necessary among the general people and for the people working in the health sector. Police department should not only focus towards the Drug User's rather they should also focuses on the dealers." Because of the short term and lack of some of the major services. eg: PHC, STI etc. Recent project was insufficient to mitigate the goal of the project". Coordination among the police and other government sector should be increased to implement program effectively. Comprehensive package was necessary to achieve the goal of the project. "There should be increment in the referral cost for the HTC and social intervention programs and nutrition was felt to be necessary for the PWIDs".

To reduce drug related harms and to reintegrate PWIDs into the society an effective programs must be implemented by the Government sector. By monitoring and evaluation of the present scenario of the PWIDs, the policy makers and the program designer must know the need of the programs to reduce the HIV infection rate in Nepal. In the recent program there was lack of the PHC services which was one of the most important components in the DICs. Furthermore, there was no any sign of program regarding the abscess management. "One of the FWIDs from Lalitpur District was unable to reach to the hospital during her abscess and was lately taken to the clinic. It was happening because there were no any programs regarding the FWIDs in Nepal". The project was found to be focused on the distribution of syringe but simultaneously also focused on the abscess management. Most of the PWIDs suffered from the abscess and were unable to reach hospital. Hence, if DIC could provide treatment for the abscess management then PWIDs will be maintained their health status.

This was the most effective programs to reduce HIV infection rate in Nepal because PWIDs were considering as the Most at Risk Populations (MAPRs). In case of the gap in the project increased the high risk behavior among the PWIDs and were stigmatized and discriminated from their surroundings and may increase the rate of HIV infections among the PWIDs and their partners. There were maximum chances of returning back towards 


\section{TARGETED INTERVENTION SERVICES FOR PEOPLE WHO ...}

the high risk behavior among the PWIDs and the effort of the project may not be achieved the goal. Due to the use of contaminated syringe PWIDs suffered from abscess and other blood borne diseases. "In the gap of the project there will be high involvement in the criminal activities among the PWIDs". Drastic changes in their behavior and their daily activities. There was a huge chance of the HIV transmission for the PWIDs and their partners." There was lack of staff motivation due to short term project".

"Free health check-up system was one of the major motivational techniques for the PWIDs. In the District Hospital if recovering leaders should be enrolled as a staff there was hope to enrolled PWIDs effectively. Furthermore, if Government could establish private unit in the hospitals for the PWIDs then the referral mechanism can implement services effectively. PWIDs always seek for the confidentiality". Government should be able to provide free treatment. E.g.: HTC, STI and PHC services. Trained counselors were necessary in the government sector. There should not be stigma and discrimination towards the PWIDs by the Government service providers. Coordination meetings among the stake holders also plays vital role to mobilize referral mechanism effective.

Firstly, government network must be able to identify the behavior, their daily activities and problems of the PWIDs. The project should conceptualize the programs according to their addictive nature of PWIDs to achieve the goal of the project. "Long term and comprehensive package was necessary to implement project effectively and mobilizing P.E actively should play vital role to identify PWIDs. In the selection of the project the most challenging factor was the low cost bidding identified as the major barrier to provide qualitative services". "The project should be able to provide travel cost and the nutrition diet to the target population for HTC clients". Requirement of the monthly coordination meeting with the local police to minimize the police harassment towards the PWIDs.

"For the staffs, training and refresher trainings were necessary to implement services effectively. One road services were necessary in the project that helps to motivate enrolled clients to express their problems. If possible shooting spots were necessary because it helps to change in their high risk behavior. There should be proper analysis on technical and financial proposal". Free treatment was necessary those who were in need 
of the treatment centers. "Government sector should coordinate with the police department to minimize the police harassment towards the PWIDs. Timely monitoring and evaluation was necessary to identify the challenges of the project and to resolve those identified challenges".

Harm reduction approaches directly related with the public health issues. Government should also focus on the qualitative services rather than to reach the target of the project. To achieve goal of the project was important part of the project but more than that was providing qualitative, long term as well as comprehensive package was most important part to reduce the drug related harms among the PWIDs. Being gap in the service was itself a challenge that increases high risk behavior among the PWIDs that results transmission of HIV and other blood borne disease. How long we should provide service wouldn't matter in case of gaps in the services impact the project into zero level.

\section{CASE STUDIES ANALYSIS}

The name used in the case study was hypothetical because of the confidentiality \& Privacy of the clients.

This was a case study conducted during the field visit in Kaski district. Mr.' A' was 21 years old. He grew up in the Pokhara city, Phulbari11. He started to use drugs in his early teens and had been injecting drugs to full fill his active addiction. He had been through addiction treatment a despite many attempts to quite, he always ended up back on drugs. Mr. 'A' became sick and tested positive for Hepatitis $\mathrm{C}$.

This was the high risk behavior case during the gap of the project. One day Mr. 'A' didn't get syringe for injecting drugs. Then he tried a lot to find a clean syringe but unable to get. He was sick and unable to handle his sickness of the injecting drugs. Thus, to kill his sickness he went to Manipal hospital in search of the syringe. In the garbage area of the hospital he found contaminated syringe. His active addition was powerful then himself. Therefore, to kill his sickness he injects the contaminated syringe to fulfill his active addiction. According to his version, "there was no any other way then to use contaminated syringe to fulfill my injecting behavior". The main reason to use such contaminated syringe was because 
of stigma and discrimination and moreover PWIDs are dehumanized by the medical stores to buy syringe.

This was only the one case study but many more were in hidden group who enforced to live their life through high-risk behavior during the gap of the project. This case study also represents not only of a single drug users but the problems among the PWIDs. It was very hard for drug user's to get syringe to fulfill their injecting behavior.

This was a case study conducted during the field visit in Kathmandu district. It was a case study of FWIDs. Mrs. B was from Kalanki with her second husband and with a daughter of her first Husband. Unfortunately, her recent husband was also from the PWIDs community. She started injecting drugs at her teen age. Now she was 27 years old. She was tested positive for Hepatitis C.

She enrolled in the OST package to quit her Injecting habits but later she was unable to quit and back to her injecting habits. After growing injecting drugs habit, one day she suffered of absences in her leg. She asked help for the treatment but was unable to get help for her abscess treatment. Furthermore, she went to hospital for the treatment but due to high cost for the treatment she was unable to receive treatment. Till now she was living her life with the abscess which was so painful and with her addiction and one daughter to feed which was so pathetic life she was living. According to her, "It was very hard to live life with abscess and there was no any treatment facility for the FWIDs". Sometime in her room, her daughter played with syringe that was so dangerous environment for her daughter living in. Sometime, dealing drugs for her addiction was another part in her life. The main reason of abscess was due to use of the contaminated syringe and sharing syringe in a group which was high risk of other blood borne disease.

This case study represented the life living by the FWIDs in Nepal. Due to poverty many of the FWIDs were unable to receive treatment. Most of the FWIDs were still hidden because of the stigma and discrimination in Nepal. In compare to male injecting drug users, female drug users were in more pathetic situation in Nepal. 


\section{CONCLUSION}

Targeted intervention program for PWIDs was the way to implement HIV prevention programs in settings with concentrated HIV epidemics. It provides prevention and care services to PWIDs population within communities by providing them with the information, skills, care support and treatment services through referral mechanism. Drug abuse was a global problem. It affects almost every country, although it was extent and characteristics differ from region to region. Drug abuse trends around the world, especially among youth, have started to converge over the last few decades. Drug-related problems include increased rates of crime and violence, susceptibility to HIV/AIDS and hepatitis, demand for treatment and emergency room visits. Therefore, Harm Reduction was the mainstay for the Injecting Drug User's. Therefore to minimize the HIV infection rate there should be more services for the current Injecting Drug User's. Moreover, we need to understand that gap in the project was the main reason for the transmission of HIV and other blood borne disease among the PWIDs in Nepal. Harm reduction programs save lives, save money and help respect, protect and fulfill the human rights of people who use drugs. Therefore, Dis- continuation of the project itself a challenging factor that hinders the Life of the PWIDs and enforces to live a pathetic lifestyle to survive this shows the continual, effective and active involvement of the professional social workers for the macro level intervention in the related area.

\section{REFERENCES}

Corrigan, P.W. (2005). On the stigma of mental illness. New York: Guilford Press.

Drug Control Policy, (2006). Retrieved form http://www. lawcommission. gov.np.

Cook., Phelan, M., Sander, G., Stone, K., \& Mrphy, F,. (2016). Harm reduction international, The case for a harm reduction decade: progress, Potential and Paradigm shifts. London:Authors.

Namuna Integrated Devlopment Council. (2017). Annual report: Author.

Central Bureau of Statistics. (2006). Hard drug users in Nepal, Kathmandu : Author. 
214 TARGETED INTERVENTION SERVICES FOR PEOPLE WHO ...

NCASC, (2010). National targeted intervention operational guidelines. Vol. 2. Author.

- - (2016/17). Press release on IBBS survey among most at risk population: Author.

Perlman, H.H. (1957). Social case work: A problem solving process. Chicago: University of Chicago Press. 